\title{
Evolution of sunspot properties during solar cycle 23
}

\author{
F. T. Watson ${ }^{1}$, L. Fletcher ${ }^{1}$, and S. Marshall ${ }^{2}$ \\ 1 School of Physics and Astronomy, SUPA, University of Glasgow, Glasgow G12 8QQ, UK \\ e-mail: f.watson@astro.gla.ac.uk; lyndsay.fletcher@glasgow.ac.uk \\ 2 Department of Electronic and Electrical Engineering, University of Strathclyde, Glasgow G1 1XW, UK \\ e-mail: s.marshall@eee.strath.ac.uk
}

Received 4 February 2011 / Accepted 21 July 2011

\begin{abstract}
Context. The long term study of the Sun is necessary if we are to determine the evolution of sunspot properties and thereby inform modeling of the solar dynamo, particularly on scales of a solar cycle.

Aims. We aim to determine a number of sunspot properties over cycle 23 using the uniform database provided by the SOHO Michelson Doppler Imager data. We focus in particular on their distribution on the solar disk, maximum magnetic field and umbral/penumbral areas. We investigate whether the secular decrease in sunspot maximum magnetic field reported in Kitt Peak data is present also in MDI data.

Methods. We have used the Sunspot Tracking And Recognition Algorithm (STARA) to detect all sunspots present in the SOHO Michelson Doppler Imager continuum data giving us 30084 separate detections. We record information on the sunspot locations, area and magnetic field properties as well as corresponding information for the umbral areas detected within the sunspots, and track them through their evolution.

Results. We find that the total visible umbral area is $20-40 \%$ of the total visible sunspot area regardless of the stage of the solar cycle. We also find that the number of sunspots observed follows the Solar Influences Data Centre international sunspot number with some interesting deviations. Finally, we use the magnetic information in our catalogue to study the long term variation of magnetic field strength within sunspot umbrae and find that it increases and decreases along with the sunspot number. However, if we were to assume a secular decrease as was reported in the Kitt Peak data and take into account sunspots throughout the whole solar cycle we would find the maximum umbral magnetic fields to be decreasing by $23.6 \pm 3.9$ Gauss per year, which is far less than has previously been observed by other studies (although measurements are only available for solar cycle 23). If we only look at the declining phase of cycle 23 we find the decrease in sunspot magnetic fields to be 70 Gauss per year.
\end{abstract}

Key words. Sun: activity - Sun: evolution - Sun: photosphere - sunspots

\section{Introduction}

Sunspots are dark areas on the solar surface and are associated with strong magnetic fields. The magnetic field inhibits the convective flow of plasma in the region and as this is the primary mechanism for heat transport at the surface, the sunspot is cooler and darker. Study of sunspots started around the early $1600 \mathrm{~s}$ although there are records of observations in China going back for 2000 years (Yau \& Stephenson 1988; Eddy et al. 1989). Since the discovery of the magnetic field in sunspots (Hale 1908) they have been a primary indicator of solar activity and detailed records have been kept. By studying the evolution of sunspot characteristics (area, field strength, etc), on timescales of days we can gain insight into their formation and dispersal, while studies on longer timescales (months and years) can reveal the longer-term behaviour of the Sun's large-scale magnetic field, naturally of great importance for constraining models of the solar dynamo. For example, the North-South asymmetry of sunspot numbers and areas is well-established and has been studied for many decades (see e.g. Carbonell et al. 1993; Zharkov \& Zharkova 2006; Carbonell et al. 2007, and references therein) and may indicate a phase lag between the magnetic activity in the northern and southern hemispheres, possibly hinting at non-linear behaviour, such as random fluctuations of the dynamo terms and strong high order terms (e.g. Ossendrijver 2003).

The sunspot cycle variation of many solar parameters is of course well established, however it was reported by Penn \& Livingston (2006) that Zeeman splitting observations of the strongest fields in sunspot umbrae show a secular decrease between 1998 and 2005, apparently without a clear cyclic variation. This goes hand-in-hand with an increase in the umbral brightness. Such a secular change, if verified, would have striking implications for the coming sunspot cycles - Penn \& Livingston (2010) suggest that if the trend continues there would be virtually no sunspots at the time of cycle 25 . It is one of the main goals of the present study to automatically examine the MDI data for such behaviour. In creating the dataset necessary to do this we also obtain and report on the cycle-dependent behaviour of sunspot areas and locations. In particular, the total projected area of sunspots present on the visible disk is of interest in solar spectral irradiance studies (Willson 1982; Pap 1985; Fligge \& Solanki 1997) where it enters as a parameter in spectral irradiance calculations.

We are fortunate now to have long and consistent series of solar observations from which such parameters can be extracted, and the computational capacity to do it automatically. Image 
processing and feature recognition/tracking in solar data is now a very active field (Aschwanden 2010), and sunspot detection is a well-defined image processing problem that has been studied by several authors (Zharkov et al. 2005; Colak \& Qahwaji 2008; Curto et al. 2008; Watson et al. 2009). It is the purpose of this article to detail some physical properties of sunspots detected in the continuum images from the SOHO/MDI instrument (Scherrer et al. 1995) and how they vary throughout solar cycle 23. We have used an image processing algorithm based on mathematical morphology (Watson et al. 2009).

The article proceeds with Sect. 2 detailing the generation of the sunspot catalogue and the results of looking at evolution in sunspot area and locations over solar cycle 23. Then, Sect. 3 details the evolution of magnetic fields in sunspots, particularly in the umbra where the fields are strongest. Finally, in Sect. 4 we finish with our discussion and conclusions.

\section{Creating a catalogue of sunspots}

In order to analyse the sunspots over solar cycle 23, the STARA (Sunspot Tracking And Recognition Algorithm) code developed by Watson et al. (2009) was used, and readers are referred there for information on the method and its testing. This is an automated system for detecting and tracking sunspots through large datasets and also records physical parameters of the sunspots detected. It involves using techniques from the field of morphological image processing to detect the outer boundaries of sunspot penumbrae. This is achieved by means of the top-hat transform which allows us to remove any limb-darkening profile from the data and to perform the detections in one step. In addition to the method given in Watson et al. (2009) the code had to be developed further to separate the umbra and penumbra of spots as we would be looking at the magnetic fields present in the umbra. When visually inspecting the data there is a clear intensity difference between the umbra and penumbra in sunspots. This difference is due to the magnetic structure of susnpots. The umbra has a higher density of magnetic flux which inhibits convection more than in the penumbral region. This causes the umbra to be cooler and therefore appear darker. However, as sunspots move towards the limb both the umbra and penumbra are limbdarkened. For this reason, we cannot use a single threshold value to define the outer edge of the umbra. The algorithm we use removes all limb darkening effects at the same time as sunspot detection, greatly increasing speed as these two steps are carried out together. This problem has been approached by other authors using different techniques, for example the inflection point method of Steinegger et al. (1997), the cumulative histogram method of Pettauer \& Brandt (1997), the fuzzy logic approach of Fonte \& Fernandes (2009), and the morphological approach of Zharkov et al. (2005). Our method begins with the sunspots (which includes umbrae and penumbrae) detected by STARA, and then produces a histogram of sunspot pixel intensities for each spot. This clusters in two peaks, the local minimum between which corresponds to the intensity value at the edge of the umbra. A similar histogram-based approach was implemented by Fonte \& Fernandes (2009) who then used concepts from fuzzy logic to assign membership to umbra or penumbra; they showed that particularly the pixel membership of the penumbra can vary significantly (tens of percent) depending on a parameter known as the membership function, but this is apparently less of a problem for low-resolution data, in which brightness variations within the penumbra are smeared out. We have not adopted such a method, but have instead identified the local minimum for each sunspot's histogram, and created a mask for umbral pixels. We

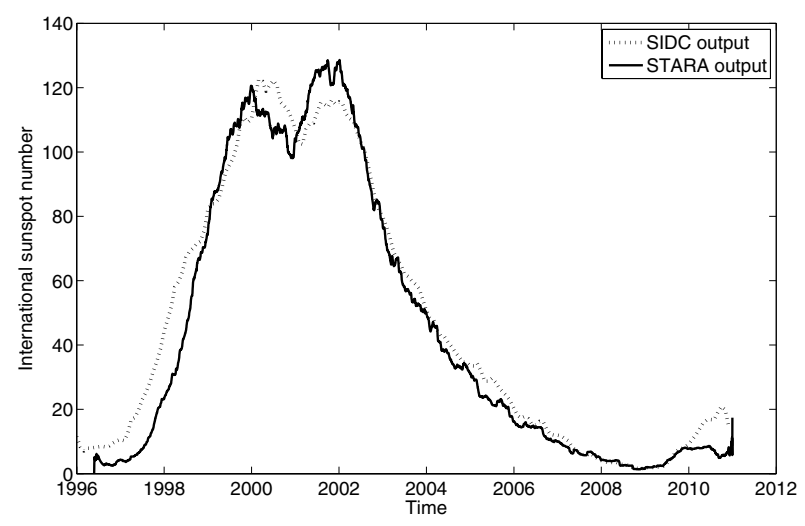

Fig. 1. The solid line shows the number of sunspots detected by the STARA code, scaled to match the magnitude of the international sunspot number near the peaks as calculated by the SIDC, shown by the dashed line.

normally find that the umbra region of sunspots has an MDI pixel value of less than 7000-8000. However, our algorithm does have the benefit of being applied consistently across the entire data series, and being able to deal with the varying intensity across the solar disk due to limb darkening which eases the problems of sunspot detection and area estimation that occur if a straightforward intensity threshold is used.

The data used in this study are taken from the MDI instrument (Scherrer et al. 1995) on the SOHO spacecraft. We use the level 1.8 continuum data as well as the level 1.8 magnetograms to analyse magnetic fields present in the spots. Our dataset uses 15 years of data and we analyse daily measurements taken at 0000 UT when co-temporal continuum images and magnetograms are recorded. The STARA code takes around $24 \mathrm{~h}$ to process the approximately 5000 days of data available to generate the sunspot catalogue used in this article and holds 30084 separate sunspot detections. The same sunspot will be detected in many different images and tracked from image to image allowing them to be associated with one another. The physical parameters obtained from this analysis are the sunspot total area and "centre of mass" location, number and area of umbrae; mean, maximum and minimum magnetic fields in the umbrae and penumbra; total and excess flux in the umbrae and penumbra and the information relating to the observation itself such as time and instrument used.

\subsection{Number of sunspots}

The trend of sunspot number throughout a solar cycle is well documented and generally rises rapidly at the start of a solar cycle before a slower decrease towards the end of the cycle. The Solar Influences Data Center (SIDC, http://www. sidc.be/ sunspot-data/) keeps records on the sunspot index and so we compare the results of our detections with the findings of the SIDC as an initial test. It must be noted that both indicators are not measuring the same thing as the international sunspot number recorded by the SIDC weights the sunspots seen in groups so that it becomes a stronger proxy for solar activity whereas STARA only gives us the raw number of observed sunspots. However, it is beneficial to see if the same trends are present. The data used here are the smoothed monthly sunspot number (SIDC-team 2010) and so our daily measurements have been treated in the same way to give a fair comparison.

In Fig. 1 we can see that both curves share several features. The STARA output has been scaled up to the same level as the 
International Sunspot Number around 2001-2003 when sunspot count rates were higher and the general trends are more important here than absolute values due to the differences in counting methods (this scaling is permissible due to the somewhat arbitrary factors present in the SIDC sunspot numbers - see Eq. (1)). We see that both datasets exhibit the same patterns of increasing and decreasing at the same time and the agreement is very good in the declining phase of the cycle. This also continues into cycle 24 shown at the right hand side of the plot with both curves rising at the same time and we will continue to track the agreement of these further into the next cycle.

The SIDC data (Clette et al. 2007), shown as a dashed line on the plot has a smooth rise up to the first maximum sometime in the year 2000 and falls before reaching a second maximum in 2002. This "double maximum" feature, separated by the "Gnevyshev gap" (Gnevyshev 1967) is also seen in the STARA output although the first maximum is weaker when compared to the second, in contrast with the SIDC data in which the first maximum is larger than the second. However, both sets of data scale well with one another after this second maximum with very little deviation and this continues from 2002 up to the current day.

The differences in the first peak, and indeed in the rise before that are most likely due to the method of counting sunspots as mentioned previously. In fact, the SIDC sunspot number is calculated using the formula

$T=k(10 g+s)$

where $T$ is the total sunspot number for that measurement, $g$ is the number of sunspot groups observed and $s$ is the number of individual sunspots observed. It is based on the assumption that sunspot groups have an average of 10 sunspots in them and so even in poor observing conditions, this would be a good substitute. The coefficient $k$ is a number that represents the seeing conditions from the observing site and is usually less than 1 .

What Fig. 1 suggests is that the SIDC observers are either detecting more sunspots than STARA in the first half of the cycle, or that they are detecting groups that have fewer than 10 sunspots in them, on average. This second explanation is more likely. Inspecting the STARA data we find it is rare to see a sunspot group with as many as ten spots in this stage of the cycle, which would account for the SIDC number being an overestimate for the actual sunspot number at this time. This in itself has interesting implications for the solar cycle, suggesting that very complex magnetic groups - and the heightened activity that accompanies them - are more likely to appear in the second part of the overall solar maximum.

\subsection{Sunspot locations}

The locations of sunspots were also recorded by the STARA code and this allows us to produce a butterfly diagram of sunspot locations. The "butterfly" shape is produced by the pattern of sunspot emergences seen in each cycle. At the start of a cycle sunspots tend to appear at high latitudes, between 20 and 40 degrees above and below the solar equator. But as the cycle progresses, the spot emergences are observed closer to the equator. The cycle then ends before the sunspots are seen to emerge at the equator and as a result of this it is very rare to see a sunspot forming within a few degrees of the solar equator. Zharkov et al. (2007) have observed a "standard" butterfly pattern in sunspot emergences in cycle 23 and our results are shown in Fig. 2.

The butterfly shape can be clearly seen as can some other features. There are gaps in 1998 as the SOHO spacecraft was

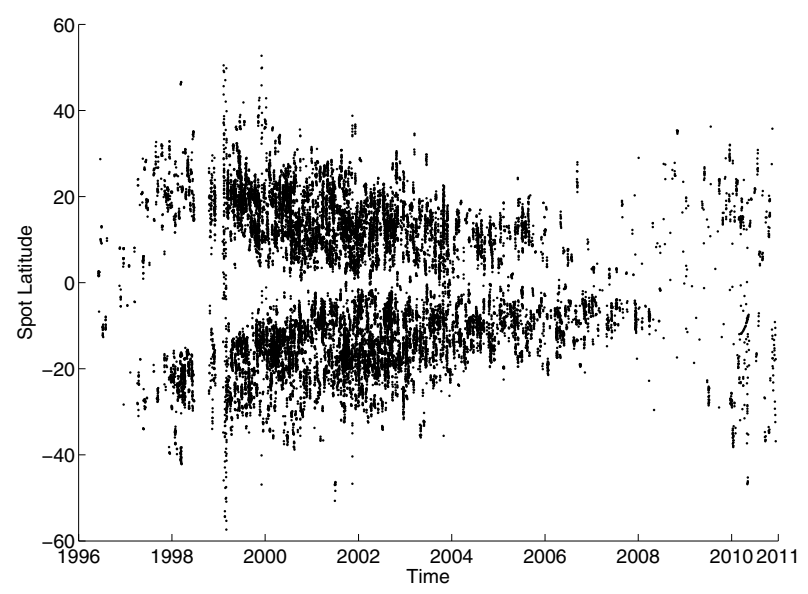

Fig. 2. The latitude of all 30084 sunspot detections from solar cycle 23 . The end of solar cycle 22 can be seen as well as the onset of cycle 24 . Note that there is a much larger "gap" between cycles 23 and 24 than between cycles 22 and 23. This confirms the lack of solar activity from mid 2008 to early 2010 .

lost for some time and no data were recorded. Also, the vertical line in early 1999 corresponds to the failure of the final gyroscope onboard and a rescue using gyroless control software. This caused the spacecraft to roll and so all data recorded at this time does not have a consistent sun orientation. These artifacts have been left in the figure (although corrected for in our subsequent analysis) to illustrate some of the potential problems with using long term data sets.

To enable the continuation of the mission the spacecraft is rotated approximately every three months to allow the high gain antenna to point at the Earth as it can no longer be moved. This means that the data are rotated and this introduces further small errors in position detection as the roll angle is not known exactly but the algorithm assumes that the data is either "north up" or "south up".

We can see from Fig. 2 that the end of solar cycle 23 exhibited asymmetric behaviour with very few spots appearing on the north hemisphere compared to the south. Hathaway (2010) shows that a north-south asymmetry in sunspot area during a cycle is very common but he also states that any systematic trend in the asymmetry during a solar cycle is found to change in the next cycle and so is not particularly useful for predictions of activity or for solar dynamo modelling. This asymmetry was studied in more detail by Carbonell et al. (1993) using a variety of statistical methods and they found that a random component was dominant in determining the trend of hemispheric asymmetry in sunspots.

\subsection{Sunspot areas}

As was the case with the number of sunspots detected, the area of the largest visible sunspot also follows the activity of the solar cycle with a clear rising phase and a slower declining phase. When calculating the area of a sunspot or umbra the number of pixels within the spot or umbral boundary is corrected to take into account the geometrical foreshortening effects that change the observed area relative to its position on the solar disk. We show this in Fig. 3. The variation is larger as sunspot sizes have a larger range than the number of spots that are present. Again, this has been smoothed to give a fair comparison to the international sunspot number calculated by the SIDC. An interesting feature of this plot is that at the start of cycle 24 there is no significant 


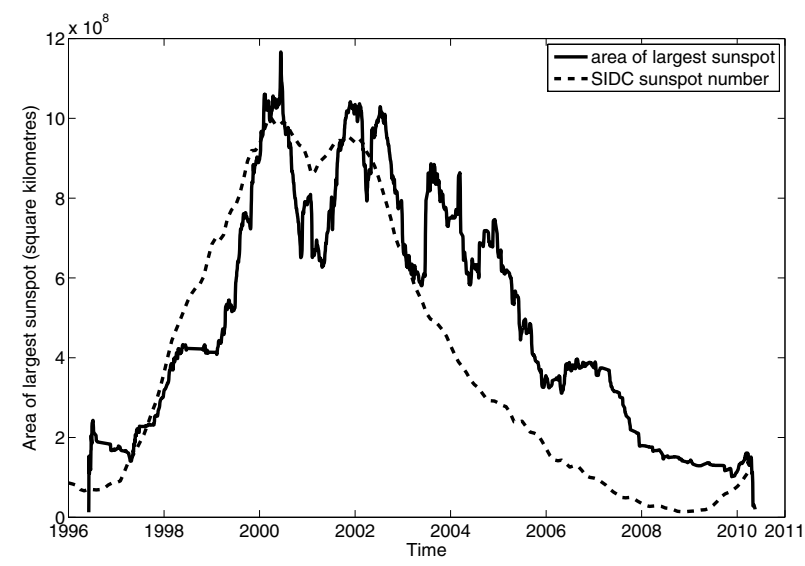

Fig. 3. The area of the largest sunspot observed is shown here, smoothed over 3 months to minimise the effect of very large sunspots and days where no spots were visible. This roughly follows the international sunspot number as well as the activity seen throughout solar cycle 23 .

increase in the areas of observed spots so we can say that there are more spots beginning to appear but the spot magnetic fields are still weak.

In addition to looking at the largest sunspot areas observed, we are also able to examine the total area of the solar surface covered by sunspots at any one time. This is shown in Fig. 4. Both the total sunspot and umbral areas are shown and, yet again, they both follow the overall trend of the solar cycle with increases and decreases at the same times. More interesting than this however, is the ratio of umbral area to sunspot area, shown in the bottom panel. We observe that the umbral area is $20-40 \%$ of the total observed sunspot area and the ratio stays within this range throughout the cycle. Even though a large variety of sunspot shapes and configurations are seen, the fractional area of associated umbra does not show high amplitude fluctuations unlike the maximum sunspot area observed - the dominant characteristic is a relatively smooth variation. Note that this does not hold for individual sunspots due to the variety of configurations seen, only to the large scale distribution of sunspots over time. There are also interesting features present, most of all the dip in the year 1999. At this time, the sunspot area is increasing more quickly than the area of the associated umbrae. This soon changes and the umbral areas start to occupy more of the sunspot again, rising by a few percent by 2004 before starting to drop off again. During the first peak in solar activity in 2000 we see that the umbra is occupying a lower fraction of the sunspot and from Fig. 1 this is when the International Sunspot Number was higher than the STARA sunspot count. This could indicate that there are sunspot groups with lower than ten sunspots present in them. This suggests that there is more space in these groups for the sunspot penumbrae to grow. In comparison to this, in the second peak of activity in 2002 we see that the fraction of sunspot area occupied by umbrae has grown and that the STARA count rate is above the International Sunspot Number. This suggests that we are seeing sunspot groups with more than ten spots in them. These would be very complex groups and so it may be the case that the sunspots have multiple umbrae present within them which would likely increase the fractional umbral area.

In Figs. 3 and 4 we show the error in the areas measured as a shaded band surrounding the line representing the data points. Estimating the errors involved is done by examining the output of the STARA algorithm. When detecting sunspots and sunspot umbrae, the centroid of the region is determined with
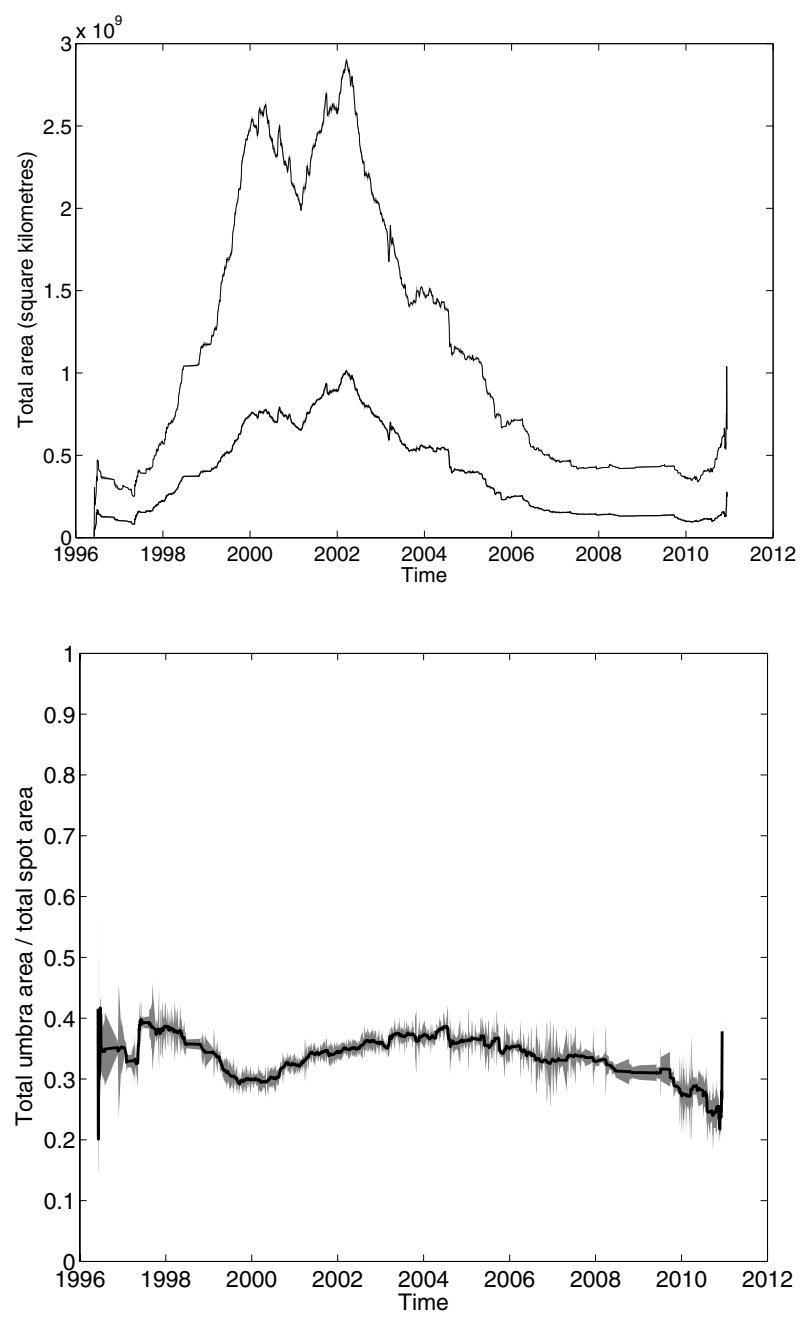

Fig. 4. Top panel: the upper line shows the total observed sunspot area and the lower line shows the total umbra area smoothed over three month periods and corrected for foreshortening effects. Only sunspots within $60^{\circ}$ of the centre of the disk were used to minimise errors from this correction. Bottom panel: the ratio of total umbral area to total sunspot area. This ratio is fairly constant, with the umbral area consiting of $30-40 \%$ of the total sunspot area and does not vary rapidly throughout the cycle. The errors are shown by the shaded area and are lower between 1999 and 2005 due to the increased number of sunspots at that time.

good accuracy. However, when defining the perimeter of the region, we believe that there is an error of 1 pixel both towards and away from the centre of the region. This means that large sunspots will have a smaller fractional error than small spots, even though the absolute value of the error will be greater for large spots.

We also show the percentage of the projected solar disk covered by sunspots from the viewpoint of the SOHO spacecraft in Fig. 5. The trend is very similar to that of the absolute total area of sunspots looked at previously. We see the fraction of the solar disk covered by sunspots rise to about $0.35 \%$ at the peak of activity in cycle 23 which is equivalent to $3500 \mathrm{MSH}$ (millionths of a solar hemisphere). This is comparable to some of the largest sunspots ever detected. There are significant short-term fluctuations in this series, in addition to the overall solar cycle variation. 


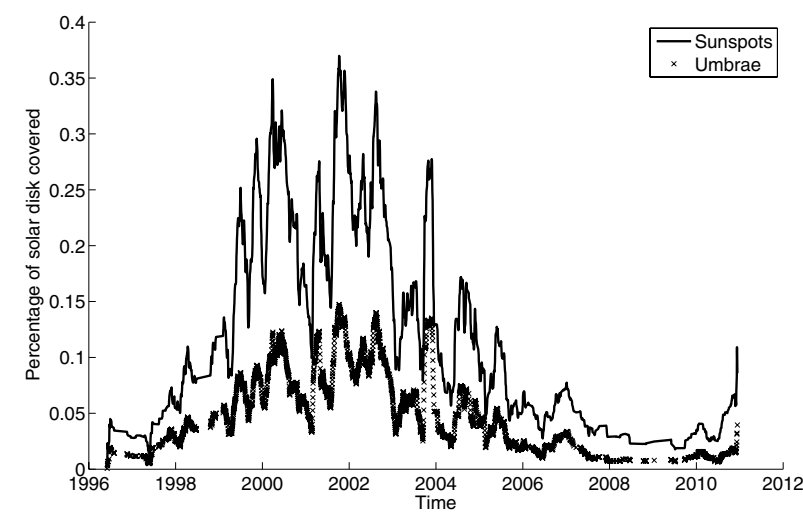

Fig. 5. We show the total sunspot (solid line) and umbra (crosses) area here as a percentage of the area of the projected solar disk. The data are smoothed over a three month period.

\section{The evolution of sunspot magnetic fields}

As the detection algorithm is directly linked with the MDI magnetograms recorded at the same time, we are also able to track the evolution of the magnetic field present in sunspots throughout the cycle. We assume that the magnetic field within sunspot umbrae is in the local vertical direction. As the MDI data only gives the line of sight magnetic field we apply a cosine correction to account for this. The amplification of magnetic field strength due to the cosine correction becomes very large as sunspots approach the limb, so making an incorrect assumption about the field being vertical can lead to vastly wrong B values at the limb. To minimise these effects we only include sunspots with a value of $\mu>0.95$ where $\mu$ is the cosine of the angle between the local solar vertical and the observers line of sight. In addition to this, the observed line of sight field is corrected with the assumption that the true field direction is perpendicular to the local photosphere. As we are looking at the strongest fields in sunspot umbrae this is a reasonable approximation.

Figure 6 shows the maximum sunspot umbral fields measured daily from 1996-2010. The first thing to notice is the spread of magnetic fields measured. We also see that the majority of measurements fall between 1500 and 3500 Gauss. It is very difficult to see any kind of trend in the data due to the spread of values but we can observe a lack of strong sunspots from 2008-2010 when the most recent solar minimum occured.

A similar study has been undertaken by Penn \& Livingston (2006) using the McMath-Pierce telescope on Kitt Peak which includes umbra measurements going further back, to 1991. The method is different as they use the Zeeman splitting of the Fe I line (1564.8nm) to infer a magnetic field strength at the location of the measurement. Measurements are made in the darkest part of the umbra, where this is identified in the image using a brightness meter. The Zeeman splitting identified at that location is used to determine the true magnetic field as the splitting of the spectral line observed is not dependent on the angle between the magnetic field and the observers line of sight. Very small spots were excluded from their dataset, as the small size of the umbra increases the risk of scattering of penumbral radiation into the umbral area, and consequent distortion of the line profile. Pore fields correspond to the range 1600-2600 G, with a mean of $2100 \mathrm{G}$. When this dataset of maximum measured umbral field is binned and averaged by year, and plotted as a function of time, a decrease is visible which can be fitted with a linear trend equivalent to around -52 Gauss per year. We repeat the analysis carried out by Penn \& Livingston (2006) on

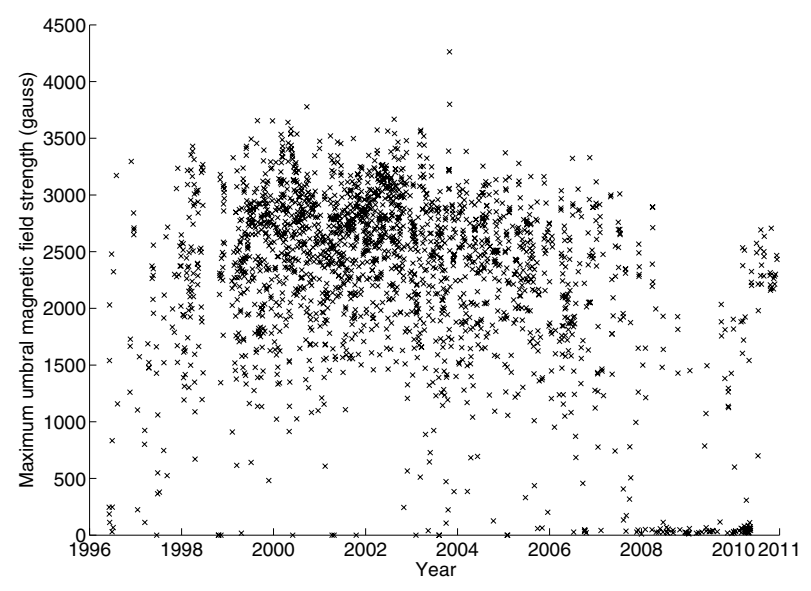

Fig. 6. Maximum sunspot umbra field from 1996-2010. Measurements are taken daily.

our dataset, both including and excluding all spots with a vertical magnetic field component below $1500 \mathrm{G}$ to minimise the possible effects of pores being included in the analysis, for a direct comparison with the Penn \& Livingston (2006) result. The results are shown in Fig. 7.

The top panel includes all sunspots detected whereas the bottom panel excludes any spots with a maximum field strength of less than 1500 Gauss. The error bars are calculated as the standard error on the mean of all measurements in the bin.

The data are in line with a picture in which the umbral fields are simply following a cyclical variation pattern, as the increases and decreases follow the international sunspot number. This cannot be confirmed with the current data and we will need to wait until the next cycle is well under way to see if the trends continues to be present. If we do a straight line fit as in Penn \& Livingston (2006), then the gradient of the best fitting line gives a decrease in umbral fields of $23.6 \pm 3.9$ Gauss per year which, although still decreasing, is a far slower decline than seen by Penn \& Livingston. Repeating the analysis excluding sunspots with fields below 1500 Gauss gives a long term decrease in field strength of $22.4 \pm 3.9$ Gauss per year. This is even further from the result they observed, although as the sunspots with fields below 1500 Gauss make up such a small fraction of the population we observe, we would not expect a significant change in the result. Other studies have also cast doubt on the long term decrease of umbral magnetic fields. The Penn \& Livingston (2006) article suggests that a decrease of 600 Gauss over a solar cycle would cause a change in mean umbral radius as a relationship between these two quantities has been shown by Kopp \& Rabin (1992) and Schad \& Penn (2010) but follow up observations by Penn \& MacDonald (2007) could not see this in their data. It has also been suggested by Mathew et al. (2007) that a small sunspot sample may introduce a bias into results if the size distribution of sunspots used is not calculated in advance.

However, the long term decline in sunspot magnetic fields does agree with the lack of an increase in sunspot area as shown in Fig. 3. If the magnetic field is now weaker than at the same time in the last cycle we would expect sunspots to be smaller and this is currently what is observed.

Interestingly, if the data from only the declining phase of the cycle (from 2000 to 2010) are used, then the maximum umbral field strengths are seen to decrease by around 70 Gauss per year which is far greater than the Penn \& Livingston (2006) study.

This then leads to the question of how valid this comparison is. In fact, instruments such as MDI and the new Helioseismic 

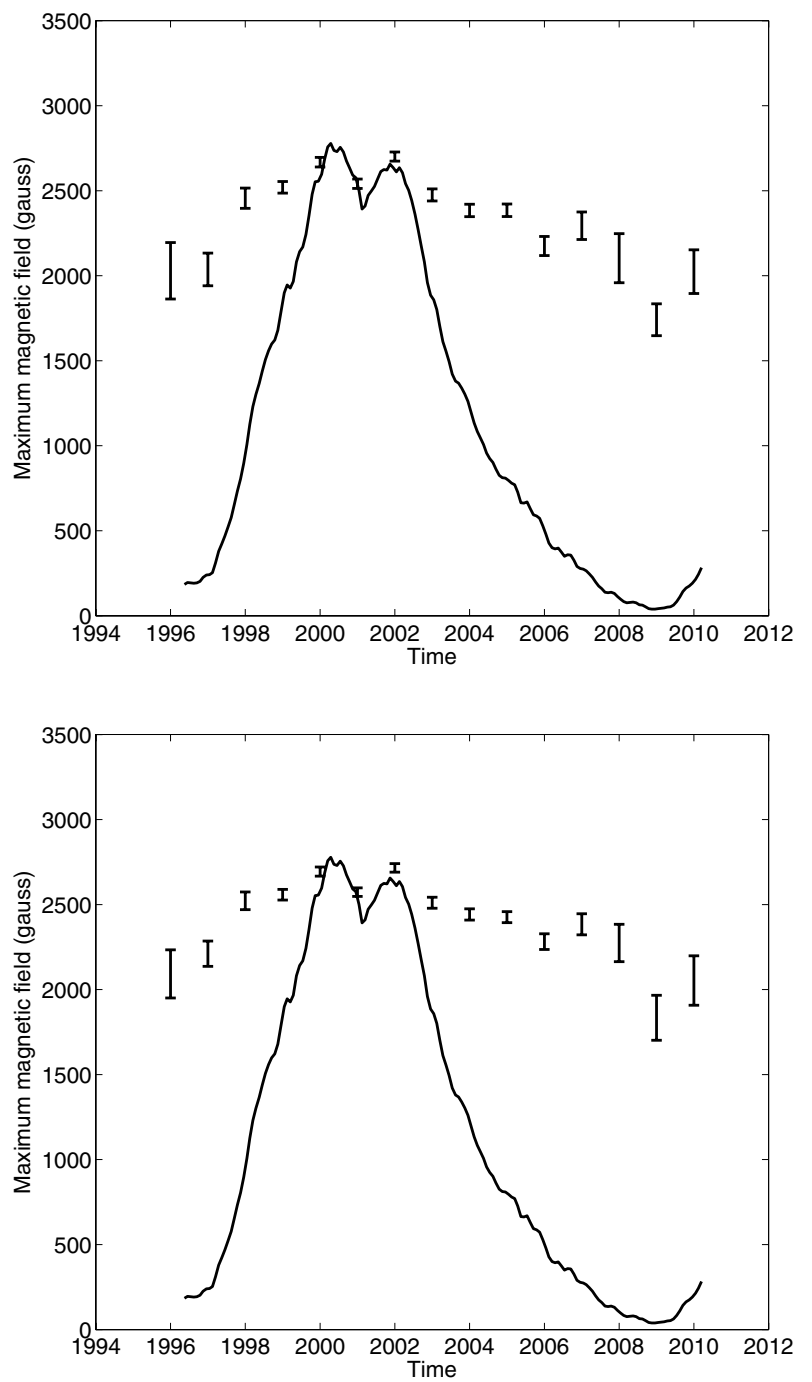

Fig. 7. The data shown in Fig. 6 have been binned by year and the mean of each bin is plotted here. Top panel: all data from Fig. 6 are included. Bottom panel: only measurements with a field above 1500 Gauss are included. The error bars correspond to the standard error on the mean. The solid line shows the evolution of the international sunspot number over the same period for reference. Assuming a linear trend gives a gradient of $-23.6 \pm 3.9$ Gauss per year and $-22.3 \pm 3.9$ Gauss per year respectively.

and Magnetic Imager on SDO do not measure the true value of magnetic field strength in a pixel. The value they return is an average magnetic field strength with a resolution determined by pixel size. However, if the filling factor of spatially unresolved magnetic elements within the pixel is close to unity, then the pixel value is a good approximation for the true line of sight magnetic field strength. This is thought to be the case deep in the umbrae of strong sunspots and so for these measurements we can say that our observations are good approximations for the true line of sight magnetic fields. In addition to this, we have only used sunspots with $\mu>0.95$ which corresponds to 18.2 degrees from solar disk centre in an effort to minimise any corrections to the magnetic field measurements but still assume that the field in the core of sunspot umbrae is perpendicular to the local photosphere.

Also, MDI has problems with saturation in magnetic field measurements with a peak value of between 3000 and 3500 Gauss depending on when the observation was made (the saturation value has lowered as the instrument degrades). This has a greater effect on measurements made at solar maximum and so has the effect of reducing the long term field strength decrease. However, this does not fully account for the discrepancy between our value of the rate of long term field decrease and that of other studies.

We have not only compared the trends seen but also the data points used in calculating these trends. The latest Livingston and Penn data is kept up to date by Leif Svalgaard and can be viewed at his own website (see www.leif.org/research). With the exception of a single data point in 1994, the Livingston and Penn yearly averages are similar to ours. Sadly, there are no yearly averages in the Livingston and Penn data between 1994 and 2001 to better compare the two studies.

\section{Discussion and conclusions}

Using a catalogue of sunspot detections created by the STARA code provides a reliable way to analyse the long term variation of certain physical parameters relating to sunspots. We found that the number of sunspots detected compared very well with the international sunspot number, even through the period of 2008-2010 when sunspot detections have been more sparse and difficult due to the decreased magnetic field strengths that are causing them. When looking at the locations of sunspots a traditional butterfly pattern is seen which also shows the end of cycle 22 as well as the period of almost no sunspots from late 2008 to early 2010 before cycle 24 started. Figure 2 also shows some of the problems of a long term observing run, such as spikes in early 1999 caused by failure of the gyroscopes onboard SOHO. In addition to this, the high gain antenna on SOHO malfunctioned in mid 2003.

The area of sunspots was then examined with the maximum spot area being first observed. The rough pattern of an initial steep rise and gradual fall associated with a solar cycle was seen but with many other features present. However, when the total observable sunspot area was plotted, a much smoother evolution was seen. The same smooth evolution was also present in the total observable umbral area. We also found that throughout the whole of solar cycle 23, if smoothed over a three month period, the area of umbra visible was between 20 and $40 \%$ of the visible sunspot area once corrections for geometric foreshortening had been applied.

We then continued to show the evolution of magnetic fields in sunspot umbrae and Fig. 6 shows the large spread of sunspot magnetic fields observed. Once the spot magnetic field data had been binned by year, a long term cyclical trend could be observed but it is yet unknown whether this is a cyclical variation around a long term linear decrease as suggested by other studies. Our data supports stronger fields near solar maximum and weaker fields at solar minimum. When compared with other similar studies, the rate of magnetic field decrease is very different and is likely due to the wide range of sunspot fields. The next solar cycle should bring a more definitive answer to the question of whether a secular trend in sunspot fields exists over multiple solar cycles. We will continue to track this for as long as SOHO still flies and also plan to incorporate data from the new Helioseismic and Magnetic Imager on the Solar Dynamics Observatory spacecraft which serves as the successor to SOHO.

Acknowledgements. F.T.W. acknowledges the support of an STFC Ph. D. studentship. This work was supported by the European Commission through the SOLAIRE Network (MRTN-CT-2006-035484) and by STFC rolling grant STFC/F002941/1. SOHO is a project of international cooperation between 
F. T. Watson et al.: Evolution of sunspot properties during solar cycle 23

ESA and NASA. We acknowledge useful discussions with M. Hendry and would like to thank our anonymous referee for their thought provoking comments. Thanks also to Leif Svalgaard for allowing us to use his plots for comparing with Livingston and Penn data.

\section{References}

Aschwanden, M. J. 2010, Sol. Phys., 262, 235

Carbonell, M., Oliver, R., \& Ballester, J. L. 1993, A\&A, 274, 497

Carbonell, M., Terradas, J., Oliver, R., \& Ballester, J. L. 2007, A\&A, 476, 951

Clette, F., Berghmans, D., Vanlommel, P., et al. 2007, Adv. Space Res., 40, 919

Colak, T., \& Qahwaji, R. 2008, Sol. Phys., 248, 277

Curto, J. J., Blanca, M., \& Martínez, E. 2008, Sol. Phys., 250, 411

Eddy, J. A., Stephenson, F. R., \& Yau, K. K. C. 1989, Quart. J. R. Astron. Soc., 30,65

Fligge, M. \& Solanki, S. K. 1997, Sol. Phys., 173, 427

Fonte, C. C., \& Fernandes, J. 2009, Sol. Phys., 260, 21

Gnevyshev, M. N. 1967, Sol. Phys., 1, 107

Hale, G. E. 1908, ApJ, 315

Hathaway, D. H. 2010, Liv. Rev. Sol. Phys., 7, 1
Kopp, G., \& Rabin, D. 1992, Sol. Phys., 141, 253

Mathew, S. K., Martínez Pillet, V., Solanki, S. K., \& Krivova, N. A. 2007, A\&A, 465,291

Ossendrijver, M. 2003, A\&A Rev., 11, 287

Pap, J. 1985, Sol. Phys., 97, 21

Penn, M. J., \& Livingston, W. 2006, ApJ, 649, L45

Penn, M., \& Livingston, W. 2010 [arXiv: 1009. 0784]

Penn, M. J., \& MacDonald, R. K. D. 2007, ApJ, 662, L123

Pettauer, T., \& Brandt, P. N. 1997, Sol. Phys., 175, 197

Schad, T. A., \& Penn, M. J. 2010, Sol. Phys., 262, 19

Scherrer, P. H., Bogart, R. S., Bush, R. I., et al. 1995, Sol. Phys., 162, 129

SIDC-team 2010, Monthly Report on the International Sunspot Number, online catalogue

Steinegger, M., Bonet, J. A., \& Vazquez, M. 1997, Sol. Phys., 171, 303

Watson, F., Fletcher, L., Dalla, S., \& Marshall, S. 2009, Sol. Phys., 260, 5

Willson, R. C. 1982, J. Geophys. Res., 87, 4319

Yau, K. K. C., \& Stephenson, F. R. 1988, Quart. J. R. Astron. Soc., 29, 175

Zharkov, S. I., \& Zharkova, V. V. 2006, Adv. Space Res., 38, 868

Zharkov, S., Zharkova, V. V., \& Ipson, S. S. 2005, Sol. Phys., 228, 377

Zharkov, S. I., Gavryuseva, E. V., \& Zharkova, V. V. 2007, Adv. Space Res., 39, 1753 